\title{
IBIS: A NEW POST-FOCUS INSTRUMENT FOR SOLAR IMAGING SPECTROSCOPY
}

\author{
F. CAVALLINI \\ INAF: Osservatorio Astrofisico di Arcetri, Largo E. Fermi, 5, 50125 Firenze, Italy \\ (e-mail:fabio@arcetri.astro.it)
}

(Received 11 November 2005; accepted 7 March 2006)

\begin{abstract}
A new instrument for solar bi-dimensional spectroscopy, the Interferometric BIdimensional Spectrometer (IBIS), has been successfully installed at the Dunn Solar Telescope of the National Solar Observatory (USA-NM) in June 2003. This instrument is essentially composed of a series of two Fabry-Perot interferometers and a set of narrow-band interference filters, used in a classic mount and in axial-mode. It has been designed to take monochromatic images of the solar surface with high spectral $(\mathcal{R} \geq 200000)$, spatial $\left(\simeq 0.2^{\prime \prime}\right)$, and temporal resolution (several frames $\left.\mathrm{s}^{-1}\right)$. IBIS has a circular field of view, $80^{\prime \prime}$ in diameter and, with suitable interference filters, it can be used in the wavelength range $580-860 \mathrm{~nm}$. The wavelength stability of the instrumental profile is very high, the maximum drift in 10 hours amounting to $\simeq 10 \mathrm{~m} \mathrm{~s}^{-1}$. In this paper the criteria used in the design and the expected instrumental characteristics are described.
\end{abstract}

\section{Introduction}

It has become increasingly evident that many quiet and active solar phenomena demand, to be properly studied, measurements over a two-dimensional field with high spectral, spatial, and temporal resolution.

The spatial resolution, of course, depends primarily on the diameter of the telescope entrance pupil as well as on atmospheric seeing. However, as far as the spectrometer is concerned, to completely exploit the telescope resolution, three conditions must be at least satisfied: i) the instrument itself must not impair the optical quality of the telescope; ii) the detector must allow a suitable spatial sampling; iii) the exposure time must be sufficiently short to reduce the effects of atmospheric distortion and allow the application of image reconstruction techniques.

Moreover, a high spectral resolving power $(\mathcal{R} \geq 200000)$ is also required, to suitably analyze narrow photospheric lines, and a high temporal resolution (several frames $\mathrm{s}^{-1}$ ) is demanded to observe rapidly evolving active phenomena. We also need a sufficiently large field of view (FOV), to easily study active regions, a sufficiently extended wavelength range, to offer a broad choice among lines with different diagnostic power, and, finally, a high wavelength stability, to provide a good reproducibility of the selected spectral points, also in long observing runs, (e.g., oscillatory phenomena). 
Unfortunately, it is difficult to simultaneously meet all these, sometimes conflicting, requirements. So, a high spectral resolving power implies a lower photon flux on the detector, forcing an increase in the exposure time so as not reduce the photometric accuracy. However, in this case, both the temporal and the spatial resolution decrease. If the exposure time exceeds some tens of milliseconds, the seeing cannot be frozen, with a perceptible reduction of the effective spatial resolution. In reality, two different methods can be used to compensate for the seeing effects: adaptive optics ( $\mathrm{AO})$ and/or some post facto techniques. $\mathrm{AO}$ allows long exposure times, but it is only effective on small FOV's, with a typical size of $\simeq 10^{\prime \prime}$. For larger areas, post facto techniques must be used (destretching, phase diversity, speckle interferometry, blind deconvolution, etc.), all nevertheless requiring images where the spatial information has not been completely lost. Exposure times sufficiently short to freeze the seeing are therefore required.

In conclusion, an imaging spectrometer, with simultaneously high spectral, spatial, and temporal resolution is a photon-starved instrument. To have therefore a photon flux per resolved element, such as to not compromise the photometric accuracy, it is mandatory that such an instrument has the largest attainable throughput and transmittance.

To this purpose, the Fabry-Perot interferometer (FPI) seems to be a good candidate: in fact, thanks to its large area and to the modern dielectric multi-layer coatings (with very low absorption coefficients), this device has both the required characteristics. Moreover, other points in favour of the FPI are its high achievable spectral resolution, similar to that of a grating spectrograph, and its rapid wavelength tuning, if piezo-scanned.

Notwithstanding, only a few multi-FPIs for ground-based solar bi-dimensional spectrometry have been built in the past. The first one was the triple-FPI of the Culgoora Observatory (Ramsay, Kobler, and Mugridge, 1970; Loughhead, Bray, and Brown 1978), which however was never really operative.

Two hybrid systems, both using a FPI in series with a tunable birefringent filter, have been built in Göttingen (Bendlin, Volkmer, and Kneer, 1992; Bendlin and Volkmer, 1995) and in Florence (IPM: Italian Panoramic Monochromator) (Cavallini, 1998). However, the main limitations of these instruments are their slow wavelength tuning and, primarily, their low transmittance, due to the birefringent filter. Recently, three new multi-etalons have been built. The first one is TESOS (Telecentric Etalon SOlar Spectrometer), a double-FPI spectrometer which, since 1997, is operating at the Vacuum Tower Telescope (VTT) on Tenerife (Kentischer et al., 1998); in Spring 2000 TESOS was upgraded to a three-etalon system (Tritschler et al., 2002). The second multi-etalon is an improvement of the original Göttingen instrument, where the tunable birefringent filter has been replaced by a second FPI; this instrument is operative now at the VTT (Koschinsky, Kneer, and Hirzberger, 2001). The third one, described in this paper, is the Interferometric BIdimensional Spectrometer (IBIS), a double-FPI recently built at the INAF - Arcetri Astrophysical Observatory, with the contribution of the Department 
of Astronomy and Space Science of the Florence University and the Department of Physics of the Roma "Tor Vergata" University. IBIS has been operating, since June 2003, at the Dunn Solar Telescope (DST) of the National Solar Observatory (USA - NM), where, since January 2005, it is one of the facilities at the disposal of all observers.

Since 2002, a further double-etalon is operating at the National Solar Observatory (Neidig et al., 2003). This instrument, built for the ISOON patrol program (Improved Solar Observing Optical Network), is used to acquire solar images in the $\mathrm{H} \alpha$ line (once per minute), in continuum (once every 10 minutes), in line-ofsight magnetic fields, and, more recently, in the $1083 \mathrm{~nm}$ He line. This multi-FPI differs however from previous instruments for its very large FOV (full disk) and the relatively small spatial $\left(\simeq 0.6^{\prime \prime}-2^{\prime \prime}\right)$ and spectral resolution $(\mathcal{R} \leq 100000)$.

\section{The Project}

\subsection{THE OPTICAL MOUNT}

The first problem to be solved in designing a multi-FPI is the choice of the most suitable optical mount. Two different mounts can be used: classic (CM) or telecentric (TM).

In CM, the FPIs are placed near an image of the entrance pupil of the telescope, where the object is at infinity. In this case the points of the final image are formed by collimated beams, incident with different angles on the interferometer plates. In TM, instead, the FPIs are placed near an object image, where the entrance pupil of the telescope is at infinity. In this case the points of the final image are formed by equal cones of rays, normally incident on the plates, and containing all the possible directions allowed by the optics (see Figure 1).

To evaluate advantages and disadvantages of each optical mount, let us suppose firstly to use ideal FPIs, ones whose plates are perfectly parallel and without defects. In CM, under these hypotheses, the transparency profile of each interferometer only depends on the spacing $(t)$, the reflectivity $(R)$ and the refraction index $(\mu)$ of the material between the plates (air in our case). Its full width at half maximum (FWHM) is given by

$$
\mathrm{FWHM}_{\mathrm{CM}}=\lambda^{2}(1-R) /\left(2 \pi \mu t R^{1 / 2}\right),
$$

while its wavelength position shifts radially towards the blue, from the center to the edge of the field, following the well-known equation

$$
\delta \lambda_{\theta}=-\lambda \theta^{2} /(2 \mu),
$$

where $\theta$ is the incidence angle. For different FPIs with the same $\mu, \delta \lambda_{\theta}$ is the same, and the overall transparency profile is simply the product of those of each interferometer. 


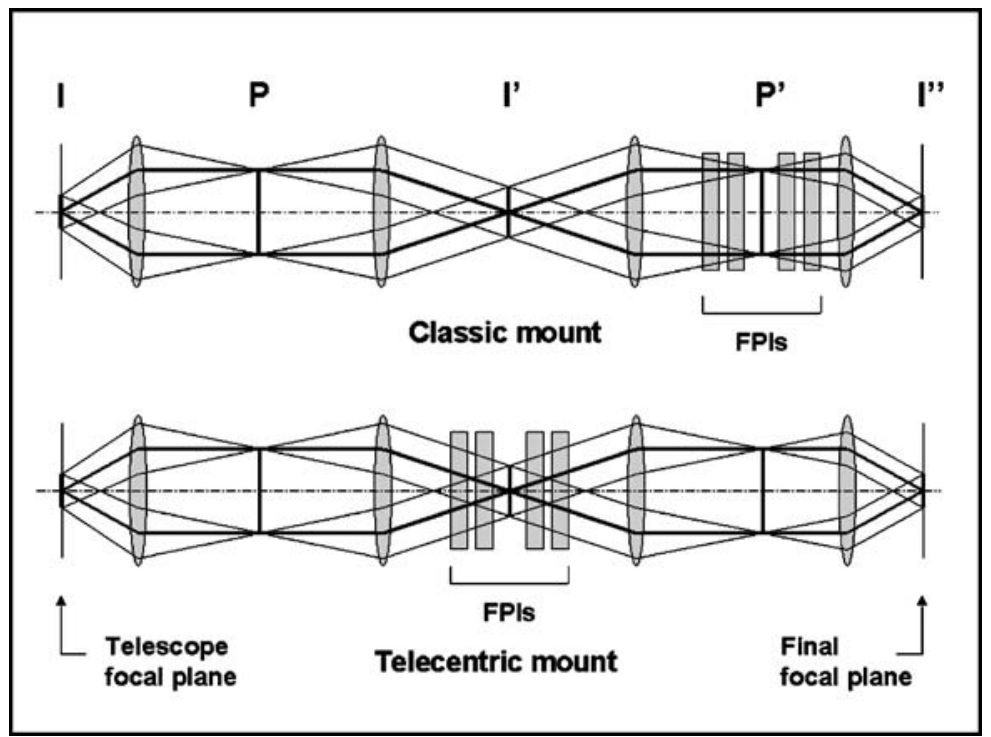

Figure 1. Schematic drawing of a double-etalon in classic and in telecentric mount. I and $\mathbf{P}$ respectively represent the sequence of images of the object and of the entrance pupil of the telescope, each one at the focus of the following lens.

For an ideal multi-FPI in CM the FOV is therefore spectrally inhomogeneous, while the spectral resolution has the maximum obtainable value. Finally, concerning the image quality, it is entirely preserved.

In TM, instead, the transparency profile of each interferometer is broadened by the finite aperture of the incident beam. Its FWHM is given by

$$
\mathrm{FWHM}_{\mathrm{TM}}=\left\{\left[\lambda^{2}(1-R) /\left(2 \pi \mu t R^{1 / 2}\right)\right]^{2}+\left[\lambda /\left(8 \mu f_{\#}^{2}\right)\right]^{2}\right\}^{1 / 2},
$$

where $f_{\#}$ is the $f$-number of the incident beam, and its wavelength position is the same at all the points of the final image. For an ideal multi-FPI in TM the FOV is therefore spectrally homogeneous, while the spectral resolution is lower than in $\mathrm{CM}$. Finally, concerning the image quality, it is reduced by a systematic effect, due to a wavelength dependent pupil apodization (Beckers, 1998; von der Lühe and Kentischer, 2000).

Both telecentric and classic mounts benefit by increasing the $f_{\#}$ of the incident beam, hereinafter meaning the inverse of the maximum incidence angle: in TM both the spectral resolution (Equation (3)) and the image quality (von der L uhe and Kentischer, 2000) are improved, while in CM the spectral inhomogeneity decreases (Equation (2)). On the other hand, a larger $f_{\#}$ also requires larger interferometers to not reduce the FOV. As a matter of fact, for small incidence angles and a constant refraction index of the medium (air, in our case), it is easy to show that, due to 
the Helmholtz-Lagrange invariant, the following relation holds for both classic and telecentric mounts:

$$
\beta=\Phi_{\mathrm{FPI}} /\left(f_{\#} \Phi_{T}\right),
$$

where $\beta$ is the field angle, and $\Phi_{\mathrm{FPI}}$ and $\Phi_{T}$ respectively are the interferometer and the telescope diameters.

We may conclude that in the ideal case the CM seems preferable, allowing the best spectral resolution and image quality, with the only disadvantage being the spectral inhomogeneity. This latter, however, can be recovered by extending the spectral scanning towards the red an amount equal to the total instrumental blue-shift (see later).

Let us suppose now to use real FPIs, ones whose plates are not perfectly flat, and let us remember that, for each interferometer, transparency peaks occur whenever

$$
2 \mu t=n \lambda \cos \theta,
$$

where $n$ is an integer. From Equation (5), we obtain:

$$
\delta \lambda=\lambda \delta t / t,
$$

showing that spacing fluctuations due to plate defects (cavity errors) produce wavelength shifts of the transparency profile.

Let us suppose that the mean spacings $\left(t_{1}, t_{2}\right)$ of the two FPIs in series are adjusted, so that two of their transparency profiles coincide at a given wavelength, when measured by integrating over the image area. If TM has been adopted, each cone of rays, corresponding to an image point, crosses both interferometers in two small areas (typical size $\simeq 1 \mathrm{~mm}$ ), where the spacings generally differ from their mean values by small amounts $\left(\delta t_{1}, \delta t_{2}\right)$. These produce the following wavelength shifts of the corresponding transparency profiles (Equation (6)): $\delta \lambda_{1}=\lambda \delta t_{1} / t_{1}$ and $\delta \lambda_{2}=\lambda \delta t_{2} / t_{2}$. If $\delta \lambda_{1} \neq \delta \lambda_{2}$, the situation is equivalent to a local detuning and the resulting transparency profile changes both in wavelength and in shape (FWHM, peak transparency, equivalent width, symmetry) in comparison with the ideal case. On the other hand, if $\delta \lambda_{1}=\delta \lambda_{2}$, the resulting transparency profile does not change at all or changes only in wavelength.

For a real multi-FPI in TM, therefore, the spectral resolution is generally lower than in the ideal case, randomly changing over the image plane, and the FOV is spectrally inhomogeneous. The wavelength fluctuations of the transparency profile can be measured and corrected (Cavallini, 1998), and the fluctuations of the equivalent width can be recovered by flat-fielding, but other shape changes (e.g., asymmetry or FWHM) are more difficult to be compensated. Finally, concerning the image quality, it is affected by the same apodization effect which is present in the ideal case.

On the other hand, if $\mathrm{CM}$ has been adopted, each collimated beam, corresponding to an image point, has a diameter not much smaller than that of each FPI and practically covers the same area of the plates. The overall transparency profiles, 
averaged over the same shift distributions due to the cavity errors, have hence the same broadened shape in all the points of the final image, while their wavelength position shows a parabolic blue-shift towards the edge of the field, as in the ideal case.

For a real multi-FPI in CM, therefore, the spectral resolution is reduced and the FOV is spectrally inhomogeneous. Finally, the image quality is impaired by the cavity errors (Ramsay, 1969).

We may conclude that the choice between classic and telecentric mount is more difficult in the real than in the ideal case. As a matter of fact, when plate defects are also considered, both mounts show reduced image quality and spectral resolution, as well as a spectrally inhomogeneous FOV. Moreover, since the manufacturer generally only specifies the maximum peak-to-peak value of the cavity errors, their effects can be only roughly estimated on the basis of some assumptions about their spatial distribution.

Some other minor aspects of the two optical mounts can also be considered, such as the possible contamination of the image in TM due to dust or coating defects on the plates, and/or due to fringing produced by any pair of plane parallel glass surfaces present in the image space where the FPIs are placed. The first problem may be solved by moving the interferometers as far as possible from the focal plane, and then removing the residual unwanted objects by flat-fielding. Concerning the fringes, they are also in principle removed by the flat-field, but only if they did not change during the observing run. This requires a constant temperature of the optical element(s) where they originate, a condition that may be difficult to satisfy.

Moreover, both in CM and in TM, to avoid the spurious images due to reflections from the rear surfaces of the plates, these latter are wedged by a suitable angle. In TM these spurious images demand a reimaging optics and an aperture stop to be eliminated; more simply, in CM, they appear separated from the principal image on the final focal plane.

On the basis of these considerations, CM has been adopted finally. From the spectroscopic point of view this mount has the advantage of a transparency profile with the same shape at all the points of the final image. Moreover, its systematic blue-shift is not difficult to correct (see later), allowing use of larger incidence angles than the TM, which needs, on the contrary, small relative apertures to achieve good image quality and spectral resolution. This implies that the CM generally allows a larger FOV (Equation (4)).

On the other hand, in CM the optical quality critically depends on the number of FPIs (see Section 2.2) and on the plate defects. Supposing that the cavity errors are essentially due to a symmetric parabolic nonuniformity, as may arise in practice in polishing or in coating the interferometer plates (Ramsay, 1969; Netterfield and Ramsay, 1974), both the optical quality and the spectral resolution may be improved by using only the inner part of the FPIs. A good compromise has been found by imposing for IBIS a maximum diameter of $35 \mathrm{~mm}$ for the pupil image: under these hypotheses, as both interferometers are $50 \mathrm{~mm}$ in diameter, the cavity errors are 
reduced by at least a factor two, from $\lambda / 100$ to $\lambda / 200$ (see Section 4.1). In this case, a Strehl ratio ranging from $0.97(580 \mathrm{~nm})$ to $0.98(860 \mathrm{~nm})$ is found for the point spread function of each interferometer (Ramsay, 1969). This is a promising result, although the Ramsay theory is limited to evaluating image quality of a single FPI, and it is not straightforward to extend it to a multi-etalon case. Moreover, the upper limit imposed on the pupil image diameter allows a sufficiently large FOV and a reasonable instrumental blue-shift. As a matter of fact, from Equations (2) and (4) we find:

$$
\delta \lambda_{\theta}=-\lambda /(8 \mu)\left(\beta \Phi_{T} / \Phi_{\mathrm{FPI}}\right)^{2},
$$

showing that for $\mu=1, \beta=80^{\prime \prime}, \Phi_{T}=762 \mathrm{~mm}$ (diameter of the DST entrance pupil) and $\Phi_{\mathrm{PPI}}=32 \mathrm{~mm}$, the instrumental blue-shift ranges from 6 to $9 \mathrm{pm}$ within the useful wavelength range $(580-860 \mathrm{~nm})$. This blue-shift is then compensated by the following procedure: i) the spectral scanning is extended an equal amount towards the red, to have the same coverage of the selected spectral intervals at the center and at the edge of the field; ii) a series of flat-field images, taken at the disk center, is used to calculate at each pixel the wavelength position of a solar spectral line $\left(\lambda_{i}\right)$ and its mean value $\left(\lambda_{M}\right)$ on the image plane; iii) a map is obtained where, at each pixel, the shift $\delta \lambda_{i}=\lambda_{i}-\lambda_{M}$ is known; iv) by fitting this map with a paraboloid, the instrumental blue-shift is finally obtained and can be then used to correct the wavelength scale of the single line profiles.

\subsection{THE NUMBER OF FPIS}

Let us consider now a series of $N$ interferometers in CM. If $\mathcal{T}_{\mathrm{IF}}(\lambda)$ and $\mathcal{T}_{i}(\lambda)$ respectively are the transmission profiles of the interference filter (IF), used as order sorter, and of the $i$-th FPI, the resulting instrumental profile $\mathcal{T}(\lambda)$ is obtained simply as the product

$$
\mathcal{T}(\lambda)=\mathcal{T}_{\mathrm{IF}}(\lambda) \prod_{i=1}^{N} \mathcal{T}_{i}(\lambda) .
$$

The next step is the choice of the number of interferometers. From the spectroscopic point of view, if the number of FPIs increases, the side unwanted interference orders are better suppressed, the spectral resolving power increases and an IF with a larger passband may be used. On the other hand, the complexity also grows and both the overall transparency and the optical quality decrease. Considering that the primary aim of this project is to build an instrument that allows us high spatial resolution, we need the highest possible transmittance and optical quality. On the basis of these considerations, we finally preferred a double rather than a triple-FP. A drawback of this solution is that very narrow-band interference filters $\left(\mathrm{FWHM}_{\mathrm{IF}} \simeq 0.3-0.5 \mathrm{~nm}\right)$ are required as order sorters (see Section 2.3). This reduces the useful wavelength range to $\simeq 0.2-0.3 \mathrm{~nm}$, sufficient, however, to analyse most Fraunhofer lines. 


\subsection{THE OPTIMUM RATIO}

Once the optical mount and the number of FPIs have been defined, the next step is the search of the optimum ratio, i.e., that ratio between the interferometer spacings, which, for a given IF, best reduces the parasitic light. This quantity is defined as the ratio between the flux outside and inside the instrumental profile, namely as

$$
P=\left[\int_{0}^{\lambda_{1}} \mathcal{T}(\lambda) \mathrm{d} \lambda+\int_{\lambda_{2}}^{\infty} \mathcal{T}(\lambda) \mathrm{d} \lambda\right] / \int_{\lambda_{1}}^{\lambda_{2}} \mathcal{T}(\lambda) d \lambda,
$$

where $\lambda_{1}$ and $\lambda_{2}$ are two wavelengths around that of the transparency peak $\left(\lambda_{0}\right)$, where $\mathcal{T}\left(\lambda_{1}\right)=\mathcal{T}\left(\lambda_{2}\right) \ll \mathcal{T}\left(\lambda_{0}\right)$. To calculate $P$, recall that the transparency profile of an ideal interferometer, illuminated by a perfectly collimated beam, is

$$
\mathcal{T}_{i}(\lambda)=T_{0}\left[1+4 R(1-R)^{-2} \sin ^{2}\left(2 \pi \mu t_{i} \cos \theta / \lambda\right)\right]^{-1}=T_{0} \mathcal{A}(\lambda) .
$$

In Equation (10), $\mathcal{A}$ is the Airy function, while $T_{0}$ is the peak transparency, namely:

$$
T_{0}=[1-A /(1-R)]^{2},
$$

where $R$ and $A$ respectively are the coating reflectivity and the absorption coefficient.

Concerning IF, a Lorentzian transparency profile has been adopted:

$$
\mathcal{T}_{\mathrm{IF}}(\lambda)=\tau_{\mathrm{IF}}\left[1+\left(2 \Delta \lambda / \mathrm{FWHM}_{\mathrm{IF}}\right)^{2 n}\right]^{-1},
$$

where $\Delta \lambda$ is the wavelength separation from the transparency peak, $n$ the number of cavities and $\tau_{\mathrm{IF}}$ the peak transparency. Inserting Equations (10) and (12) in Equation (8), the resulting profile is finally obtained as

$$
\begin{aligned}
\mathcal{T}(\lambda)= & \tau_{\mathrm{IF}}\left[1+\left(2 \Delta \lambda / \mathrm{FWHM}_{\mathrm{IF}}\right)^{2 n}\right]^{-1} \times \\
& \times T_{0}^{2} \prod_{i=1}^{2}\left[1+4 R(1-R)^{-2} \sin ^{2}\left(2 \pi t_{i} / \lambda\right)\right]^{-1},
\end{aligned}
$$

where $\mu=1$ (air between the plates), $\theta=0$ (normal incidence) and the same $A$ and $R$ have been assumed for both interferometers.

By chosing for IF a $0.3 \mathrm{~nm}$ passband $\left(\mathrm{FWHM}_{\mathrm{IF}}=0.3 \mathrm{~nm}\right)$, two cavities $(n=2)$ and $\tau_{\mathrm{IF}}=0.30$, we have (Equation (12)) $\mathcal{T}_{\mathrm{IF}} \simeq 10^{-5}$ for $\Delta \lambda= \pm 2 \mathrm{~nm}$. $P$ has been therefore evaluated by assuming $\lambda_{1}=-2 \mathrm{~nm}$ and $\lambda_{2}=2 \mathrm{~nm}$ in Equation (9).

As shown in Figure 2a, the $P$ vs. ratio is a function with a series of minima and maxima. In particular, referring to Figure $2 \mathrm{~b}, P$ decreases with increasing $\lambda$ (solid-dotted line), $R$ (solid-dot-dashed line), and decreasing $t$ (dashed-solid line), while the ratio values, corresponding to the relative minima and maxima, do not change. To reduce the parasitic light, the useful wavelength range has been therefore chosen partly in the visible, partly in the near infrared $(580-860 \mathrm{~nm})$. A ratio of 0.277 has been then chosen, corresponding to the smallest of the $P$ relative 

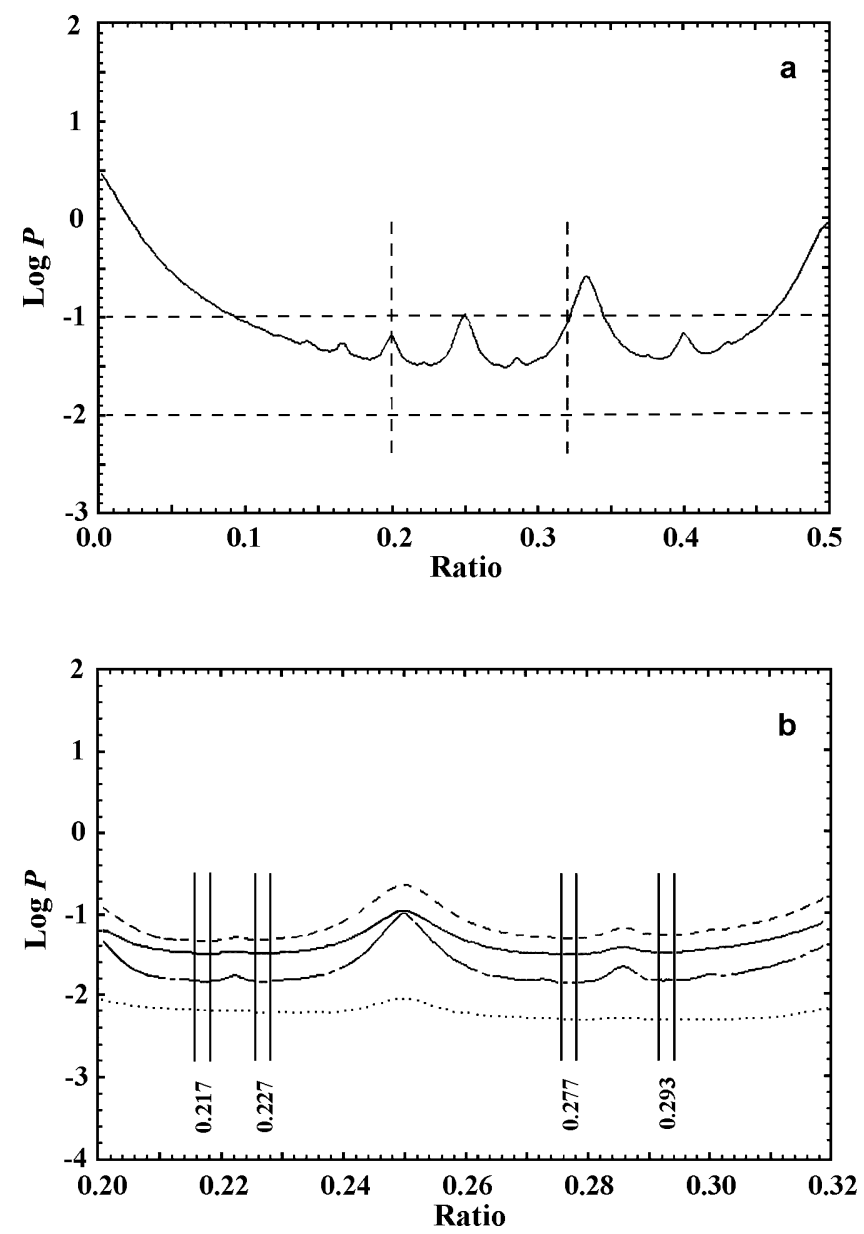

Figure 2. (a) Parasitic light $v s$. ratio for a double-etalon in classic mount, under the following conditions: wavelength: $580 \mathrm{~nm}$; coating reflectivity $(R)$ : 0.9 ; plate defects: $\lambda / 100$; spacing $(t)$ of one FPI: 2 $\mathrm{mm}$. A two-cavity interference filter with a FWHM $=0.3 \mathrm{~nm}$ has been also assumed. Ratios between 0.5 and 1.0 have been omitted, because in this range the relative minima of the parasitic light monotonically increase. The two vertical dashed lines define a range where the deepest minima can be found, while the two horizontal ones indicate $1 \%$ and $10 \%$ parasitic light. (b) Parasitic light $v s$. ratio within the range defined in (a), under different assumptions. Solid line: $t=2.0 \mathrm{~mm}, R=0.90, \lambda=580 \mathrm{~nm}$; dotted: $t=2.0 \mathrm{~mm}, R=0.90, \lambda=860 \mathrm{~nm}$; dashed: $t=2.5 \mathrm{~mm}, R=0.90, \lambda=580 \mathrm{~nm}$; dot-dashed: $t=2.0 \mathrm{~mm}, R=0.95, \lambda=580 \mathrm{~nm}$. The four relative minima within the range considered are shown by their corresponding ratio values and by a pair of vertical lines, showing the ratio uncertainty due to the spacing accuracy of $\pm 1 \mu \mathrm{m}$, claimed by the manufacturer for each interferometer.

minima, except for the dashed line (minimum at 0.217), which however is the least favorite candidate, corresponding to the largest $P$ values.

Moreover, thanks to the decrease of $P$ with increasing wavelength, for the interference filters a $\mathrm{FWHM}_{\mathrm{IF}}=0.3$ and $0.5 \mathrm{~nm}$ has been respectively adopted in 
TABLE I

Interference Filter Characteristics.

\begin{tabular}{ll}
\hline Manufacturer & Barr Associates, Inc. \\
Clear aperture $(\mathrm{mm})$ & 50 \\
Wavefront tolerance & $\lambda / 8$ over $25 \mathrm{~mm}$ \\
Cavity number & 2 \\
Blocking & $10^{-5}(200 \mathrm{~nm}-1.2 \mu)$ \\
FWHM (nm) & $0.3(580-750 \mathrm{~nm})$, \\
& $0.5(750-860 \mathrm{~nm})$ \\
Available ranges $(\mathrm{nm})$ & $0.2(580-750 \mathrm{~nm})$, \\
& $0.3(750-860 \mathrm{~nm})$, \\
Peak transparency & $\simeq 0.3$ \\
Peak wavelengths $(\mathrm{nm})$ & 589.6 \\
& 630.2 \\
& 709.0 \\
& 722.4 \\
& 854.2 \\
\hline
\end{tabular}

the ranges $580-750$ and $750-860 \mathrm{~nm}$. The primary characteristics of the selected interference filters are shown in Table I.

\subsection{THE SPACING AND THE REfleCtIVITY}

Once the optimum ratio has been found, the best spacing can be looked for. To this end, recall that the spectral resolving power $(\mathcal{R})$ is a relevant instrumental parameter depending on the spacing. For a single ideal interferometer in CM (Equation (1)):

$$
\mathcal{R}=\lambda / \mathrm{FWHM}_{\mathrm{CM}}=[\lambda(1-R)]^{-1} 2 \pi \mu t R^{1 / 2} .
$$

Two opposing requirements must be therefore envisaged: because with increasing spacing both parasitic light and spectral resolving power increase (Section 2.3 and Equation (14)), the best spacing will be a compromise between a low $P$ and a high $\mathcal{R}$. Moreover, because with increasing wavelength both parasitic light and spectral resolving power decrease (Section 2.3 and Equation (14)), these two quantities have been respectively calculated at the shortest $(580 \mathrm{~nm})$ and at the longest $(860 \mathrm{~nm})$ wavelengths used, while the broadening of the transparency profile, due to cavity errors, has been evaluated as described in Section 4.1. Finally, $\mathcal{R}$ and $P$ have been plotted in Figure 3 versus the spacing of the thickest FPI, for $R$ varying from 0.90 to 0.95 . This figure seems to suggest that the highest coating reflectivity is the best choice, providing the highest $\mathcal{R}$ and the lowest $P$. We have to consider, however, that with the increasing reflectivity, the overall maximum transmittance of the two interferometers (Equations (11) and (13)) and the image quality (Ramsay, 

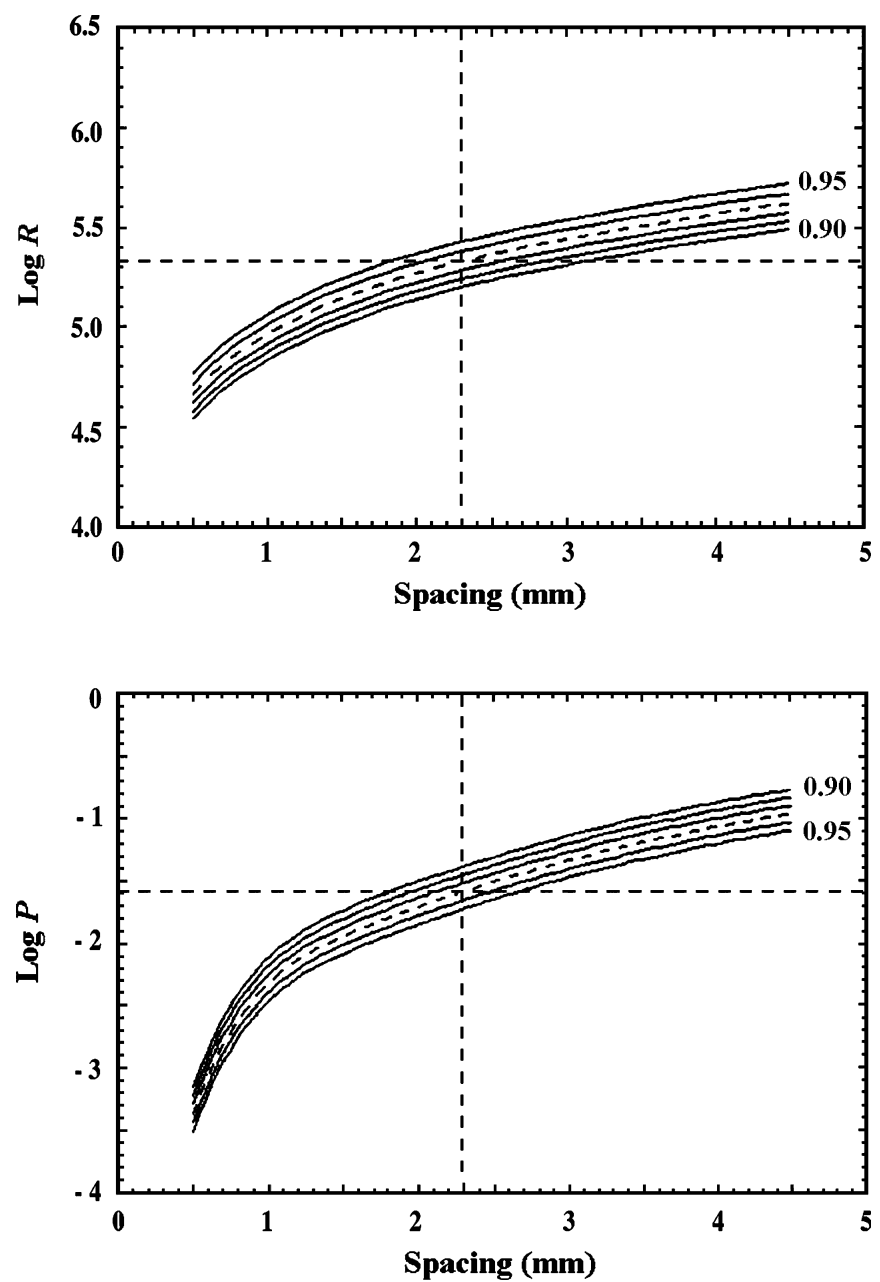

Figure 3. Abscissae: spacing of the thickest FPI. Ordinates: spectral resolving power $(\mathcal{R})$ at $860 \mathrm{~nm}$ and parasitic light $(P)$ at $580 \mathrm{~nm}$, for a plate reflectivity ranging from 0.90 to 0.95 , by steps of 0.01 . Dashed lines refer to a reflectivity of 0.93 .

1969) decrease, while the ghost relative intensity increases (see Section 2.5). This suggests using the less reflective coating, compatible with the spectral resolution and the parasitic light.

Going back to Figure 3, it may be seen that, assuming $R=0.93$ and $t=2.3 \mathrm{~mm}$ (vertical dashed line), a spectral resolving power $\mathcal{R} \geq 200000$ and a parasitic light $P \leq 2.5 \%$ are obtained.

Therefore, a pair of interferometers with spacings $t=2.300$ (FPI 1) and $0.637 \mathrm{~mm}$ (FPI 2) and a coating reflectivity $R=0.93$ have been chosen, as the best compromise between transparency, ghosts, parasitic light, image quality and spectral resolution. 
TABLE II

Fabry-Perot Interferometer Characteristics.

\begin{tabular}{ll}
\hline Manufacturer & IC Optical Systems \\
Type & ET50 FS \\
Clear aperture & $50 \mathrm{~mm}$ \\
Plate spacings & $2.300,0.637 \mathrm{~mm}$ \\
Wedge angle & $20^{\prime}$ \\
Coating & Multilayer broadband \\
Wavelength range & $580-860 \mathrm{~nm}$ \\
Reflectance & 0.93 \\
Absorption coefficient & 0.002 \\
Cavity errors (nominal) & $\lambda / 150$ (after coating) \\
Setting time & $\simeq 20 \mathrm{~ms}$ \\
\hline
\end{tabular}

The primary characteristics of both interferometers are shown in Table II.

\subsection{THE GHOSTS}

Up to this point, we have implicitly supposed that both interferometers are used in axial-mode (the plates normal to the optical axis), and we have not considered the effects of the reflected radiation. In reality, a collimated raybundle incident on a FPI produces both a transmitted and a reflected beam. The transmission profile of an ideal interferometer has been already given (Equation (10)), while its reflection profile is (Vaughan, 1989):

$$
\mathcal{R}_{i}(\lambda)=(1-A)[1-\mathcal{A}(\lambda)]+\mathcal{A}(\lambda) A^{2} R /(1-R)^{2} .
$$

Both in classic and in telecentric mounts, the inter-reflections between two interferometers in series produce ghost images at the final focal plane that must be accuratly evaluated. Let us consider therefore an IF with a transparency profile $\mathcal{T}_{\mathrm{IF}}$, followed (or preceded) by two FPIs with transparency and reflectivity $\mathcal{T}_{1}, \mathcal{R}_{1}$ and $\mathcal{T}_{2}, \mathcal{R}_{2}$, respectively. If the relative distance between the interferometers is much larger than their plate spacings, the FPIs can be considered "decoupled" (Steel, 1967) and incoherent addition of intensities may be assumed for the inter-reflected radiation. In this case it is easy to show that the ghost profile $\mathcal{G}(\lambda)$ is given by

$$
\mathcal{G}(\lambda)=\mathcal{T}_{\mathrm{IF}}(\lambda) \mathcal{T}_{1}(\lambda) \mathcal{T}_{2}(\lambda)\left[1 /\left(1-\mathcal{R}_{1}(\lambda) \mathcal{R}_{2}(\lambda)-1\right)\right] .
$$

Moreover, if $\mathcal{T}(\lambda)$ is the transparency profile of the principal image (the first transmitted one), we have (Equation (8)):

$$
\mathcal{T}(\lambda)=\mathcal{T}_{\mathrm{IF}}(\lambda) \mathcal{T}_{1}(\lambda) \mathcal{T}_{2}(\lambda) .
$$


By comparing $\mathcal{G}(\lambda)$ and $\mathcal{T}(\lambda)$, it may be seen that the former dominates the latter everywhere, save near the transparency peak, producing extended wings, that significantly reduce the spectral resolution.

Moreover, if we observe an object with a flat spectrum, the flux associated with its image is proportional to the equivalent width of the instrumental profile, defined as

$$
\mathcal{W}=\int_{\lambda_{1}}^{\lambda_{2}} \mathcal{T}(\lambda) \mathrm{d} \lambda
$$

where $\lambda_{1}=-2 \mathrm{~nm}$ and $\lambda_{2}=2 \mathrm{~nm}$ (Section 2.3). Therefore, if $\Phi_{G}$ and $\Phi_{P}$ are the fluxes corresponding to the ghosts and to the principal image respectively, we have:

$$
\Phi_{G} / \Phi_{P}=\int_{\lambda_{1}}^{\lambda_{2}} \mathcal{G}(\lambda) \mathrm{d} \lambda / \int_{\lambda_{1}}^{\lambda_{2}} \mathcal{T}(\lambda) \mathrm{d} \lambda=\mathcal{W}_{G} / \mathcal{W}_{P}
$$

It may be shown that $\Phi_{\mathrm{G}} / \Phi_{\mathrm{P}}$ increases with decreasing wavelength and increasing reflectivity, and that, in particular, for the two IBIS interferometers $(R=0.93)$, $\Phi_{G} / \Phi_{P}$ changes from $0.32(860 \mathrm{~nm})$ to $0.52(580 \mathrm{~nm})$. We may conclude therefore that the inter-reflections so seriously affect both the imaging and the spectroscopic quality, that it is mandatory to eliminate them or to reduce their effects to acceptable values.

A generally adopted solution consists in tilting one or both interferometers at a small angle, sufficient to allow the ghost images to clear the field (Loughhead, Bray, and Brown, 1978; Kentischer et al., 1998; Koschinsky, Kneer, and Hirzberger, 2001). If both interferometers are equally rotated around the same axis, but in opposite directions, the required tilt angle is

$$
\alpha \geq 1 /\left(4 f_{\#}\right) \text {. }
$$

Since generally large $f_{\#}$ are used $\left(f_{\#} \simeq 100\right)$, small tilt angles are required $\left(\alpha \simeq 0.1^{\circ}\right)$, sufficient however to produce serious secondary effects.

In CM a consequence of the tilt is a detuning of the two FPIs, increasing from the tilt axis towards the edge of the field, which produces an increasing asymmetry and a decreasing $\mathcal{W}$ of the instrumental profile. In comparison with the no-tilt case, the transparency is the same along the tilt axis, but decreases normally to this, reaching a minimum at the extremes of the perpendicular diameter. This produces an inhomogeneous darkening of the FOV, resulting in a general loss of instrumental transmittance, which can be evaluated as

$$
D(\lambda)=\left[\int_{\mathrm{FOV}} \mathcal{W} \mathrm{d} S-\int_{\mathrm{FOV}} \mathcal{W}_{\mathrm{T}}(P) \mathrm{d} S\right] / \int_{\mathrm{FOV}} \mathcal{W} \mathrm{d} S
$$

where $\mathrm{d} S$ is a surface element, and $\mathcal{W}, \mathcal{W}_{T}(P)$ respectively are the equivalent width without and with tilt, this latter depending on the image point considered $(P)$. For IBIS $\left(f_{\#}=110\right)$ a tilt angle $\alpha= \pm 0.13^{\circ}$ should be adopted to avoid the ghosts; 
this would produce an overall darkening, decreasing with increasing wavelength, ranging from $D=0.51(580 \mathrm{~nm})$ to $D=0.39(860 \mathrm{~nm})$.

In TM and in the ideal case, each cone of rays finds instead the same detuning situation in crossing the two tilted FPIs. The instrumental profile is therefore the same at each image point, but it is strongly asymmetric and with a reduced equivalent width. The result is a homogeneous darkening of the field, equivalent to an overall loss of instrumental transmittance, which, in this case, can be computed as

$$
D(\lambda)=\left(\mathcal{W}-\mathcal{W}_{\mathrm{T}}\right) / \mathcal{W}
$$

Evaluating Equation (22) for IBIS, we find again $D=0.51(580 \mathrm{~nm})$ and $D=0.39$ $(860 \mathrm{~nm})$, confirming that, for the same tilt angle, the loss of flux through the two FPIs does not change for different optical mounts. In reality, from the optical point of view, the situation at the pupil, in TM, is the same as that at the image, in $\mathrm{CM}$, and vice versa. In TM, however, due to the inhomogeneous darkening of the pupil, the tilt not only produces an asymmetric instrumental profile and a loss of instrumental transparency, but also an apodization effect, causing a further asymmetric broadening of the instrumental point spread function.

We may conclude therefore that, if the tilting is an effective method to eliminate the ghost images, it generally produces such serious secondary effects, both in classic and in telecentric mounts, that a different solution must be looked for.

Going back to the Equations (16) and (17), it may be shown that, if the IF does not follow or precede the two FPIs, but it is placed between them:

$$
\mathcal{G}(\lambda)=\mathcal{T}_{\mathrm{IF}}(\lambda) \mathcal{T}_{1}(\lambda) \mathcal{T}_{2}(\lambda)\left\{\left[1-\mathcal{T}_{\mathrm{IF}}^{2}(\lambda) \mathcal{R}_{1}(\lambda) \mathcal{R}_{2}(\lambda)\right]^{-1}-1\right\}
$$

and

$$
\mathcal{T}(\lambda)=\mathcal{T}_{\mathrm{IF}}(\lambda) \mathcal{T}_{1}(\lambda) \mathcal{T}_{2}(\lambda)
$$

By inserting Equations (23) and (24) in Equation (19), and calculating again $\Phi_{\mathrm{G}} / \Phi_{\mathrm{P}}$ for different $\tau_{\mathrm{IF}}$ values of the IF transparency peak, we find that, for $\tau_{\mathrm{IF}}=0.30$, $\Phi_{\mathrm{G}} / \Phi_{\mathrm{P}} \leq 0.015$ over all the useful wavelength range. Moreover, in this case, by comparing Equation (23) and Equation (24), it may be seen that $\mathcal{G}(\lambda)$ is everywhere negligible in comparison with $\mathcal{T}(\lambda)$.

Because $\tau_{\mathrm{IF}} \simeq 0.30$ is the typical peak transparency of the narrow-band IFs selected as order sorters for IBIS (see Table I), the solution finally adopted was to insert the filters between the two interferometers (Cavallini et al., 2000). In this way the ghost effects on the imaging and on the spectroscopy are reduced to acceptable values, and both interferometers can be used in axial-mode, without affecting the shape of the instrumental profile and, most importantly, without any transparency loss. 


\section{The Instrument}

\subsection{THE PRINCIPAL OPTICAL PATH}

IBIS has been installed at the DST on an optical bench, feed by a high-order AO system. This essentially is formed by a tip/tilt corrector, a Shack-Hartmann wavefront sensor ( 76 subapertures, 2500 frames s$^{-1}$ ), and a deformable mirror (97 actuators) described by Rimmele et al. (2004).

Figure 4 schematically represents the IBIS layout, where the principal optical path is shown by the solid line. At the exit of the high-order AO, the telescope primary image is at infinity and a pupil image is formed near the first folding mirror $\mathrm{m} 1$, at the focus of the transfer lens L0. This lens and two further mirrors $(\mathrm{m} 2, \mathrm{~m} 3)$ form the solar image on the field stop (FS) of the instrument, $21.3 \mathrm{~mm}$ in diameter, corresponding to $80^{\prime \prime}$ on the Sun. Three lenses (L1, L2, L3) and a folding mirror (M1) successively collimate the solar and the pupil image. After L3, the two FPIs, used in axial-mode and in classic mount and, between them, a filter wheel (FWH) carrying a hole, a dark slide and five interference filters (see Table I). To avoid unwanted effects from interferometer tilts (see Section 2.5), the orthogonality to the optical axis must be secured for both FPIs. To accomplish this, each interferometer can be rotated on two perpendicular axes and it is mounted on an independent translating stage, allowing it to be removed from the optical path. Before any observing run, with the sunlight feeding the instrument, a pair of pellicle beamsplitters (BS2) are inserted into the principal optical path. The first one is at $45^{\circ}$ with respect to the optical axis, while the second one is perpendicular to it and at the focus of the L2 lens, where two images are formed: the first one by L2 (direct image), the second one by L3, after reflection on one of the FPIs (reflected image). Both of these images are reflected by the first pellicle beamsplitter and a folding mirror (M4) onto a TV camera (TV2). The procedure for determining the interferometer perpendicularity is as follows. First, FPI 1 is removed from the optical path, the open position is selected in the filter wheel, and FPI 2 is rotated around the two allowed axes, so as to overlap the direct and the reflected image. Then, FPI 1 is reinserted into the optical path and the same procedure is repeated. This method allows us to adjust the perpendicularity of each interferometer to the optical axis with an accuracy of $\pm 10^{\prime \prime}$. Moreover, a diaphragm $\mathrm{D}$, placed in front of FPI 1 and centered on the incoming beam (just a few millimeters larger in diameter) can be used as reference for the position of the optical axis on the interferometer plates.

Going back to Figure 4, a fourth lens (L4) and two further folding mirrors (M2, M3) form a solar image, $6.85 \mathrm{~mm}$ in diameter, on a CCD camera (CCD 1) (see Table IV). As the overall FOV is $80^{\prime \prime}$ and the FWHM of the telescope point spread function is $0.19^{\prime \prime}$ at $580 \mathrm{~nm}, 418$ is the maximum number of resolved elements. Assuming two pixels per resolved element, a detector with at least $836 \times 836$ pixels is required. In our case the solar image is inscribed in a square area measuring 


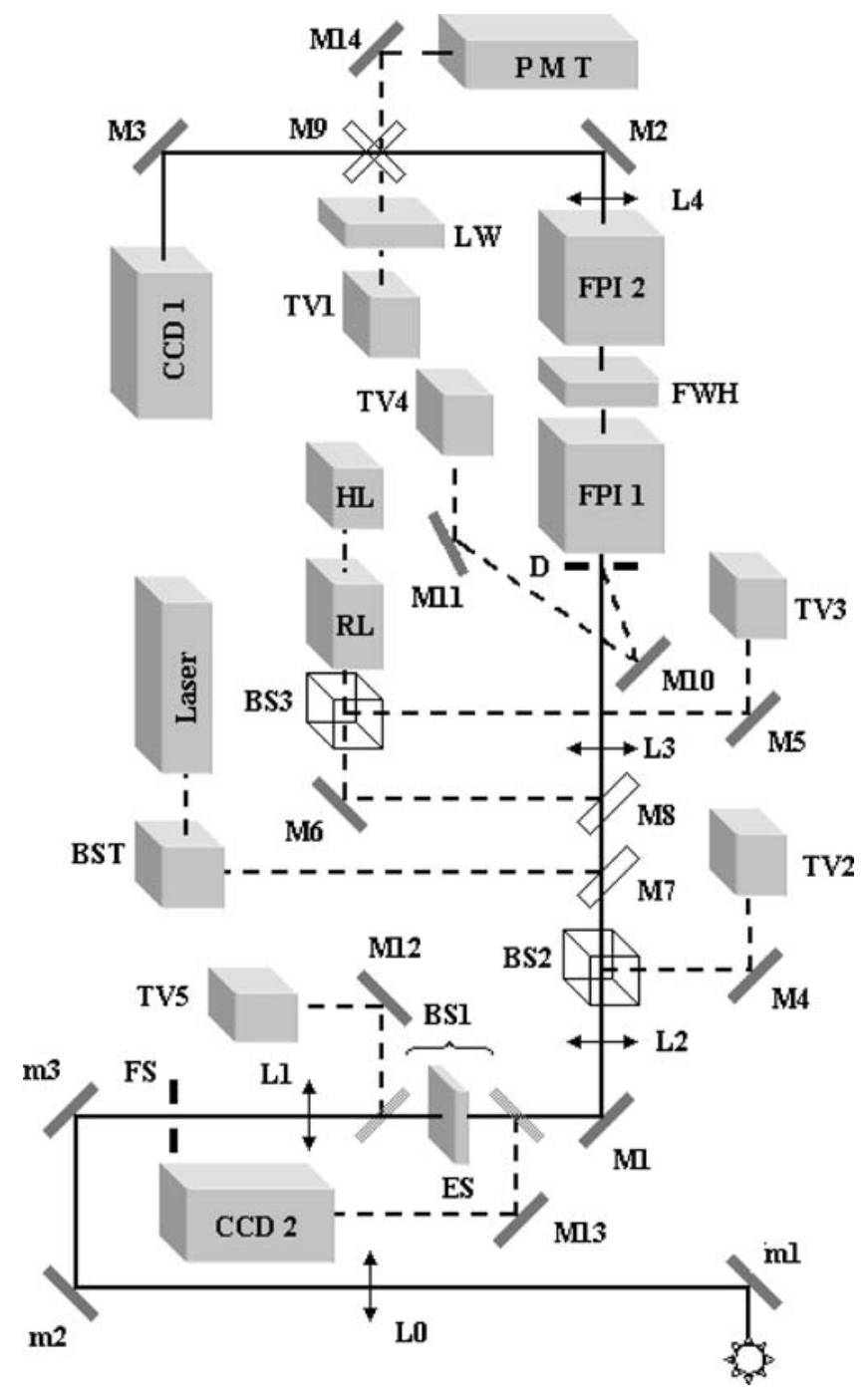

Figure 4. Schematic drawing of the instrumental layout. The solid line represents the principal optical path, while the secondary ones are shown by dashed lines. The moveable optical components, which can be inserted or extracted from the optical path, are represented as transparent objects. The meaning of the labels is as follows. BS: beamsplitter; BST: beam steering; CCD: CCD camera; ES: electronic shutter; FPI: Fabry-Perot interferometer; FS: field stop; FWH: filter wheel; HL: halogen lamp; L: lens; LW: lens wheel; M, m: mirror; PMT: photomultiplier; RL: relay lenses; TV: TV camera; W: window.

$1007 \times 1007$ pixels on a CCD with $1024 \times 1024$ pixels. This implies an image scale of $0.08^{\prime \prime}$ pixel $^{-1}$ (at least 2.4 pixels per resolved element), such as to completely exploit the telescope spatial resolution. 


\subsection{THE REFERENCE PATH}

This secondary path originates from two beamsplitters (BS1) placed between L1 and L2 (see Figure 4), in a space where the solar image is at infinity. Each of them is a BK7 plane-parallel window, anti-reflection coated on both surfaces, reflecting a small amount of the incoming light $(\leq 0.5 \%)$ to a TV (TV5) and to a CCD camera (CCD 2) (see Table IV) through two folding mirrors (M12 and M13). An electronic shutter (ES), placed between the two beamsplitters in a pupil space, simultaneously controls the exposure time for CCD 1 and CCD 2. The thickness of each beamsplitter $(12.7 \mathrm{~mm})$ is such that the secondary pupil image, coming from its rear surface, is sufficiently far from the principal one to be eliminated by means of a suitable diaphragm. Both TV5 and CCD 2 are equipped with a photographic zoom lens, allowing the adjustment of the image scale, an interference filter $(\lambda=$ $720 \mathrm{~nm}, \mathrm{FWHM} \simeq 10 \mathrm{~nm}$ ) and a pair of linear polarizers, to adjust the light level. TV5, continuously showing the selected solar region, is used to monitor the solar and atmospheric conditions. CCD 2 allows one to take, simultaneously with CCD 1 , broad-band images of the same FOV, which can be used as a transparency reference and/or for post facto procedures, to correct the seeing effects.

\subsection{THE TUNING PATH}

The initial tuning between the two interferometers and the selected interference filters is obtained by using a halogen lamp (HL). Its output is kept constant to better than $1 \%$ by a light intensity control system for a sufficiently long time such that no other reference is required. The goal is to faithfully reproduce the optical situation when the Sun is observed, but with a source with a flat spectrum. To this purpose, the lamp light is concentrated on a flashed opal diffuser, and a suitable system of relay lenses (RL) provides an image of the diffuser with the same size and $f_{\#}$ as the solar one produced by L2. This image can be put on the L3 focus by means of a fixed (M6) and of a moveable (M8) folding mirror. The perpendicularity of the lamp light to the interferometers is then verified by inserting in the optical path BS3, a pair of pellicle beamsplitters identical to BS2 (see Section 3.1). The direct and the reflected images can be seen thanks to the folding mirror M5 and the TV camera TV3, while the position of the beam on the diaphragm D is shown by TV4, through M10 and M11, as a bright ring. By adjusting the orientation of M6 and M8, it is possible to simultaneously overlap the two images and to make the ring symmetrical, securing in this way the perpendicularity and the centering of the incident beam. Once these two conditions are verified, the lenses L3 and L4, the mirrors M2 and M14, and the moveable mirror M9 finally form an image of the diffuser on the photochatode of a photomultiplier (PMT). Tuning is then performed by keeping FPI 2 at a fixed voltage and measuring the PMT signal, while scanning FPI 1. The voltage where the maximum signal is found corresponds to a tuning situation, namely to the coincidence of one interference order of FPI 1 with one of 
FPI 2. A further scanning, performed with both interferometers tuned, allows one to find the peak transparency of the selected interference filter.

\subsection{THE LASER PATH}

This secondary optical path is used to verify and to adjust the parallelism of the interferometer plates. To this purpose, the beam of a frequency-stabilized $\mathrm{He}-\mathrm{Ne}$ laser is sent through a beam steering system (BST) near the edge of a circular rotating diffuser. The result is a small bright spot, with a rapidly changing speckle pattern, which simulates a monochromatic incoherent source. This spot illuminates, at short distance, a second flashed opal diffuser ( $\simeq 8 \mathrm{~mm}$ in diameter), an image of which can be then put, by means of the moveable mirror M7, at the focus of the L3 lens.

The two lenses L3 and L4, the fixed mirror M2, and the moveable mirror M9 finally send the laser light to LW. This is a wheel, carrying three different lenses, respectively forming on TV1 an image of the diffuser and of the FPI 1 and FPI 2 plates.

As a first step, FPI 2 is removed from the optical path and a suitable lens is selected on the LW, showing on TV1 the diffuser image. Its monochromatic light, crossing FPI 1, produces a ring system, the inner part of which can be seen with superimposed the diffuser circular edge. By adjusting M7 to center the ring system on the diffuser contour, normal incidence of the laser light on the interferometer plates is obtained. By selecting a suitable lens on the LW, TV1 shows the FPI 1 plates, lighted by the laser. Due to the wavelength fluctuations of the transparency profile produced by spacing and parallelism errors, the plate image generally shows both random and systematic brightness inhomogeneities. By changing the applied voltage, so that one interference order moves back and forth in wavelength on the laser line, and adjusting, at the same time, the parallelism conditions to obtain an image changing as homogeneously as possible, the best parallelism condition is finally obtained. The same procedure can be then repeated for FPI 2, by exchanging the two FPIs on the optical path, and by selecting a third suitable lens on the LW.

\section{The Expected Instrumental Characteristics}

\subsection{THE SPECTRAL RESOLVING POWER}

To correctly calculate the spectral resolving power, the broadening of the instrumental profile, due to the cavity errors, must be properly evaluated. To this purpose, if incoherent addition of intensities is assumed, the transparency profile can be obtained as the convolution between that of an ideal FPI and the distribution function of the wavelength shifts due to the plate defects Vaughan, 1989). The peak-to-peak 
value of these, expressed as $\lambda_{0} / p\left(\lambda_{0}=632.8 \mathrm{~nm}, p=150\right.$ after coating $)$, is the only information provided by the manufacturer. We conservatively assumed $p=100$ and supposed the cavity errors as being due to a symmetric parabolic nonuniformity (see Section 2.1). The corresponding shift distribution, in this case, is a rectangular function, with a width $\lambda \lambda_{0} /(p t)$. Under these hypotheses, the overall instrumental profile can be calculated and a spectral resolving power is found, ranging from 200000 to 270000 .

\subsection{THE EXPOSURE TIME AND THE TEMPORAL RESOLUTION}

To evaluate the expected exposure time (a relevant instrumental parameter if high spatial resolution is wanted), let us estimate firstly the photoelectron flux on the detector. This may be written as

$$
\Phi\left(\mathrm{e}^{-} \mathrm{s}^{-1} \mathrm{pixel}^{-1}\right)=H^{2} D^{2} \tau_{T} \tau_{\mathrm{O}} \tau_{\mathrm{IF}} \lambda f_{\lambda} Q_{\lambda} \mathcal{W} e^{-K_{\lambda} / \cos z}\left(h c \alpha^{2}\right)^{-1},
$$

with the following meaning of the symbols. $H\left(\operatorname{arcsec}\right.$ pixel $\left.^{-1}\right)$ : image scale on the detector; $D(\mathrm{~cm})$ : diameter of the telescope entrance pupil; $\tau_{\mathrm{T}}, \tau_{\mathrm{O}}$ and $\tau_{\mathrm{IF}}$ : transparency of the telescope, of the IBIS optics and of the interference filter, respectively; $f_{\lambda}\left(\mathrm{erg} \mathrm{cm}^{-2} \mathrm{~s}^{-1} \mathrm{~nm}^{-1}\right.$ ): solar flux outside the Earth's atmosphere in the continuum between the lines; $Q_{\lambda}\left(\mathrm{e}^{-}\right.$photon $\left.^{-1}\right)$ : detector quantum efficiency; $\mathcal{W}(\mathrm{nm})$ : equivalent width of the instrumental profile; $K_{\lambda}$ : exponential absorption coefficient of the Earth's atmosphere; $z$ (degrees): Sun zenith distance; $\alpha$ (arcsec): solar angular diameter, and $h c / \lambda\left(\mathrm{erg}_{\text {photon }}{ }^{-1}\right)$ : photon energy.

If we demand a signal to noise ratio $\mathrm{S} / \mathrm{N} \geq 100$, a minimum of $10^{4} \mathrm{e}^{-}$pixel $^{-1}$ is required, namely an exposure time

$$
t(\mathrm{~ms}) \geq 10^{7} / \Phi \text {. }
$$

$\Phi$ has been then evaluated by taking $f_{\lambda}$ and $K_{\lambda}$ from Allen (1985), and $Q_{\lambda}$ from the quantum efficiency curve of the detector (Kodak KAF-1400). Moreover, $D=76.2$ $\mathrm{cm}$ (DST entrance pupil diameter), $\tau_{\mathrm{IF}}=0.30, H=0.08^{\prime \prime}$ pixel $^{-1}$ (see Table IV), and $z=45^{\circ}$ have been assumed. To evaluate $\tau_{\mathrm{O}}$, the following expression has been then used:

$$
\tau_{\mathrm{O}}=T_{L}^{4} T_{\mathrm{BS}}^{2} T_{W}^{8} T_{P_{1}}^{2} T_{P_{2}}^{2} R_{M}^{3},
$$

where $T_{\mathrm{L}}=0.988, T_{\mathrm{BS}}=0.981, T_{\mathrm{W}}=0.989, T_{\mathrm{P}_{1}}=0.986$, and $T_{\mathrm{P}_{2}}=0.983$ respectively are the transparency of each lens, beamsplitter, window, first and second plate of the IBIS FPIs. All of the surfaces of these optical elements have an antireflection (AR) coating, optimized in the range 580-860 $\mathrm{nm}$. Its reflectivity, as well as the glass internal absorption, have been considered in calculating the overall transparency. Moreover, $R_{\mathrm{M}}=0.980$ has been assumed for the reflectivity of each mirror (protected Ag). Inserting these values in Equation (27), we find $\tau_{\mathrm{O}}=0.742$. Finally, the shortest allowed exposure times $\left(t_{i}\right)$ have been evaluated by means 


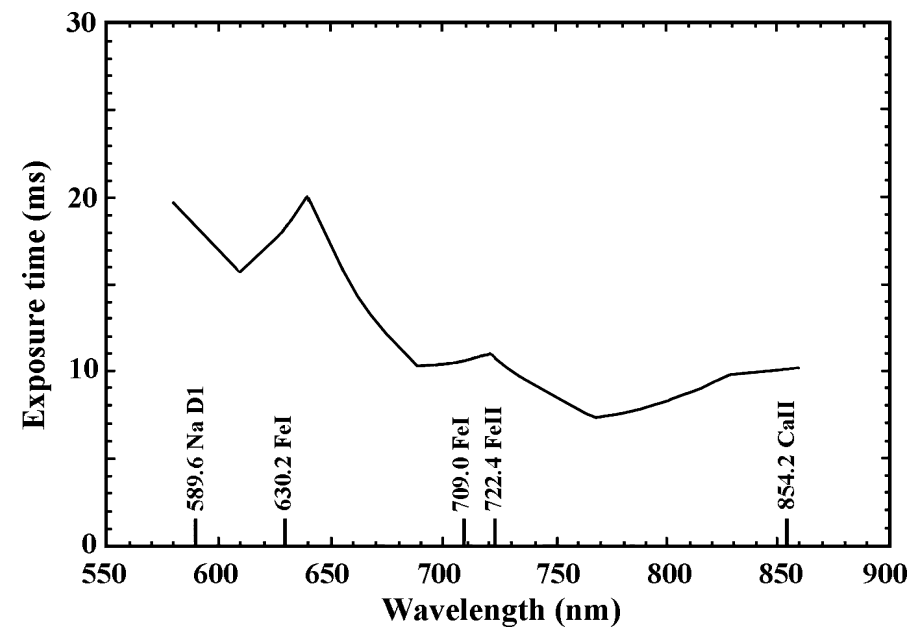

Figure 5. IBIS exposure time $v s$. wavelength, as calculated assuming $\mathrm{S} / \mathrm{N}=100$, maximum spatial resolution $(1024 \times 1024$ pixels $)$, interference filter's transparency $=30 \%$, zenith distance of the Sun $=45^{\circ}$, and telescope transparency $=100 \%$. On the abscissae: the wavelengths of the observable solar lines are shown.

of Equations (25) and (26) in the ideal case of a perfectly transparent telescope $\left(\tau_{\mathrm{T}}=1\right)$. The results are shown in Figure 5 and in the second column of Table III, for the wavelengths of the available interference filters. However, to obtain the effective exposure times $\left(t_{\mathrm{e}}\right)$, the transparency of all the optics preceding the instrument must be evaluated. To this purpose, the following expression has been used, where the contributions of the telescope, of the AO system, and of the tranfer optics ( $\mathrm{m} 0, \mathrm{~m} 1, \mathrm{~m} 2$ and $\mathrm{L} 0$ in Figure 4 ) have been all included:

$$
\tau_{T}=T_{L s}^{2} T_{L c} T_{B S} T_{W}^{2} R_{\mathrm{pAg}}^{6} R_{\mathrm{pAl}}^{2} R_{\mathrm{Al}}^{3} .
$$

Here $T_{L s}$ and $T_{L c}$ respectively are the transparency of a lens with a standard AR coating $(400-700 \mathrm{~nm})$ and with a custom AR coating $(580-860 \mathrm{~nm}) . T_{B S}$ and

TABLE III

IBIS Exposure Times.

\begin{tabular}{llll}
\hline $\begin{array}{l}\text { Peak wavelengths of the } \\
\text { interference filters }(\mathrm{nm})\end{array}$ & $t_{i}(\mathrm{~ms})$ & $\tau_{T}$ & $t_{\mathrm{e}}(\mathrm{ms})$ \\
\hline 589.6 & 16 & 0.43 & 37 \\
630.2 & 18 & 0.38 & 47 \\
709.0 & 11 & 0.34 & 32 \\
722.4 & 11 & 0.33 & 33 \\
854.2 & 11 & 0.22 & 50 \\
\hline
\end{tabular}


$T_{W}$ respectively are the transparency of each beamsplitter and window, while $R_{\mathrm{pAg}}$, $R_{\mathrm{pAl}}$ and $R_{\mathrm{Al}}$ are the reflectivity of mirrors respectively protected $\mathrm{Ag}$, protected Al, and unprotected $\mathrm{Al}$.

The calculated $\tau_{\mathrm{T}}$ and $t_{\mathrm{e}}$ values are reported in Table III, showing that the low transparency of the optics in front of the instrument sensibly increases the exposure times. In any case, the expected $t_{\mathrm{e}}$, ranging from 32 and $50 \mathrm{~ms}$, seems to be sufficiently short to freeze most of the seeing. Finally, the temporal resolution can be evaluated by considering the time required for the wavelength setting ( $\simeq 20 \mathrm{~ms}$ ), the exposure $(\leq 50 \mathrm{~ms})$, the readout $(300 \mathrm{~ms}$ at $5 \mathrm{MHz})$, and the storing $(\simeq 30 \mathrm{~ms})$ of each image $\left(1024 \times 1024\right.$ pixels). An acquisition rate of $\simeq 2.5$ frames s ${ }^{-1}$ can be therefore expected.

\subsection{The tUning AND the WAVELENGTH STABiLity}

As the instrument is essentially formed by two FPIs in series, their wavelength stability is critical, not only to warrant a good reproducibility of the selected spectral points on long observing runs, but also to avoid any detuning which could change the shape of the resulting instrumental profile. A detuning of $0.28 \mathrm{pm}$ at $580 \mathrm{~nm}$ has been assumed as the maximum permitted, producing an asymmetry of $\simeq 0.1 \mathrm{pm}$ and a decrease of $\simeq 0.5 \%$ of $\mathcal{W}$, namely of the overall instrumental transmittance. At longer wavelengths less strict requirements can be adopted.

On the other hand, the wavelength stability of each interferometer depends only on the stability of its cavity optical length ( $\mu t$ in Equation (5)). For each of the adopted FPIs, the plate parallelism and geometrical spacing $(t)$ are controlled by a close-loop electronic system (CS100), using five capacitance sensors and three piezo-electric actuators. The CS100, however, is sensitive to changes of the dielectric constant of the air and to thermal effects of the capacitor pillars and of some electronic components. Moreover, the refractive index $(\mu)$ of the air between the plates changes, due to any variation of the ambient pressure, temperature, or humidity. To eliminate the environmental effects on the capacitance sensors and on the cavity optical length, each interferometer is enclosed in a sealed cell.

The temperature sensitivity of the capacitor pillars produces a drift of $0.28 \mathrm{pm}^{\circ} \mathrm{C}^{-1}$ for FPI 1 and $0.27 \mathrm{pm}{ }^{\circ} \mathrm{C}^{-1}$ for FPI 2 . This effect has been reduced by means of a thermostatic system, similar to that used for the IPM FPI (Cavallini, 1998), able to mantain each interferometer at $\simeq 38^{\circ} \mathrm{C}$ within $\pm 5 \times 10^{-3}{ }^{\circ} \mathrm{C}$. In this way a maximum drift of $\pm 1.4 \times 10^{-3} \mathrm{pm}$ (for both positive or negative shifts), and a maximum detuning of $2.8 \times 10^{-3} \mathrm{pm}$ (for opposite shifts) have been obtained.

Moreover, the CS100 temperature sensitivity $\left(50 \mathrm{pm}^{\circ} \mathrm{C}^{-1}\right)$, producing a drift of $1.3 \times 10^{-2} \mathrm{pm}{ }^{\circ} \mathrm{C}^{-1}$ for FPI 1 and $4.6 \times 10^{-2} \mathrm{pm}{ }^{\circ} \mathrm{C}^{-1}$ for FPI 2 , has been compensated by means of a rough temperature control $\left( \pm 0.1^{\circ} \mathrm{C}\right)$, obtained by controlling the speed of the cooling fan of each CS100. In this way the maximum 
drift and the maximum detuning have been respectively reduced to $\pm 3.0 \times 10^{-3} \mathrm{pm}$ and $5.9 \times 10^{-3} \mathrm{pm}$.

The largest source of detuning is however the CS100 digital resolution (12 bits), allowing the change of the plate spacing by discrete steps of $0.49 \mathrm{~nm}$. Due to the different spacings of the two FPIs, the corresponding wavelength steps $(\Delta \lambda)$ are (Equation (6))

$$
\Delta \lambda_{1} / \Delta \lambda_{2}=t_{2} / t_{1} .
$$

This implies that the minimum wavelength step (see Table IV) is imposed by the interferometer with the smallest spacing (FPI 2), while the maximum possible detuning is one half of the FPI 1 minimum wavelength step, namely $6.2 \times 10^{-2} \mathrm{pm}$ at $580 \mathrm{~nm}$. This is the worst situation, when the peak wavelength of the FPI 2 order used is in the middle of two admitted wavelength positions of one FPI 1 interference order.

Therefore, the maximum expected detuning, when all of the possible effects are considered, amounts to $7 \times 10^{-2} \mathrm{pm}$, well below the maximum admitted value of $0.28 \mathrm{pm}$, while the maximum expected drift amounts to $\pm 4.4 \times 10^{-3} \mathrm{pm}$, or $\pm 2.3 \mathrm{~ms}^{-1}$, in velocity units.

However, some tests of long-term wavelength stability, performed on a similar interferometer with an equivalent thermostatic system Cavallini 1998), showed a larger drift, amounting to $\simeq 10 \mathrm{~ms}^{-1}$ in 10 hours, which has been conservatively assumed also for IBIS. This is equivalent to assuming that all of the thermal instabilities evaluated before amount to $2 \times 10^{-2} \mathrm{pm}$ for each FPI. In this case, therefore, the maximum expected detuning, when the CS100 digital resolution is also considered, amounts to $0.1 \mathrm{pm}$, while the maximum expected drift amounts to $\pm 0.01 \mathrm{pm}$, or $\pm 5.2 \mathrm{~ms}^{-1}$, in velocity units. Both drift and detuning are larger under this assumption, but small in absolute value; the detuning, in particular, is still below the maximum admitted value of about a factor three.

Finally, the wavelength stability of the interference filters must be also considered. To reduce the parasitic light and to avoid an excessive loss of transparency, a narrow range $(\Delta \lambda)$ around the peak wavelength of each interference filter is used, where the transparency is larger than $85 \%$ of the peak. This implies a $\Delta \lambda \simeq 0.2$ $\mathrm{nm}$ for the filters with a $0.3 \mathrm{~nm}$ passband, and a $\Delta \lambda \simeq 0.3 \mathrm{~nm}$ for those with a $0.5 \mathrm{~nm}$ passband (Equation (12)). Since the maximum transparency gradient is $0.7 \% \mathrm{pm}^{-1}$ at $\pm 0.1 \mathrm{~nm}$ from the peak wavelength of the filters with a $0.3 \mathrm{~nm}$ passband (Equation (12)), and since they have a temperature sensitivity of $\simeq 2 \mathrm{pm}^{\circ} \mathrm{C}^{-1}$, the filter wheel has been closed with two glass windows and the temperature inside has been stabilized within $\pm 0.1^{\circ} \mathrm{C}$. This achieves a wavelength stability of $\pm 0.2 \mathrm{pm}$ of the interference filters, corresponding to a maximum transparency variation of $\pm 0.14 \%$. 
TABLE IV

IBIS Characteristics.

\begin{tabular}{|c|c|}
\hline Wavelength range & $580-860 \mathrm{~nm}$ \\
\hline Spectral resolving power & $200000-270000$ \\
\hline Wavelength drift & $\leq 10 \mathrm{~ms}^{-1}$ in $10 \mathrm{~h}$ \\
\hline $\begin{array}{l}\text { Wavelength blue-shift on } \\
\text { the image plane (radial) }\end{array}$ & $6-9 \mathrm{pm}$ \\
\hline Field of view (circular) & $80^{\prime \prime}$ \\
\hline Wavelength setting time & $\simeq 20 \mathrm{~ms}$ \\
\hline Minimum wavelength step & $0.45-0.66 \mathrm{pm}$ \\
\hline \multirow[t]{2}{*}{ Monochromatic camera } & Roper PentaMAX \\
\hline & $\begin{array}{l}1317 \times 1035 \text { square pixels } \\
6.8 \mu \mathrm{m} \text { in size. } \\
\text { Dyn. range: } 12 \text { bits } \\
\text { Data rate: } 5 \mathrm{MHz}\end{array}$ \\
\hline \multirow[t]{5}{*}{ Broad band camera } & Dalsa CA-D7-1024T \\
\hline & $1024 \times 1024$ square pixels \\
\hline & $12 \mu \mathrm{m}$ in size. \\
\hline & Dyn. range: 12 bits \\
\hline & Data rate: $10 \mathrm{MHz}$ \\
\hline Image scale & $0.08^{\prime \prime}$ pix $^{-1}$ (2.4-3.6 pix/r.e.) \\
\hline Exposure time & $32-50 \mathrm{~ms}$ \\
\hline ( $\mathrm{S} / \mathrm{N}=100$ in the solar continuum) & $(1024 \times 1024$ pixels $)$ \\
\hline Acquisition rate including: & $\simeq 2.5$ frames s $^{-1}(1024 \times 1024$ pixels $) ;$ \\
\hline $\begin{array}{l}\text { wavelength setting, } \\
\text { exposure, frame reading, } \\
\text { storing }\end{array}$ & $\simeq 4$ frames $\mathrm{s}^{-1}$ (binning $2 \times 2$ ) \\
\hline
\end{tabular}

\section{Summary}

The IBIS instrumental characteristics, shown in Table IV, seem to meet the initial requirements well. The spectral and the temporal resolution are satisfactory, as well as the useful wavelength range, the FOV, and the wavelength stability. Concerning the spatial resolution, the image scale allows a suitable sampling and the exposure time is sufficiently short to use post facto image restoring techniques. At first glance, the monochromatic images seem of good quality, showing, when compared with simultaneously taken broad-band images, a comparable spatial resolution and only a slight decrease in contrast. However, without knowing the real cavity errors and 
their spatial distribution, it is difficult to quantitatively evaluate the effective image quality. This latter, as well as other instrumental characteristics which need to be directly measured, will be the subject of a following paper.

\section{Acknowledgements}

Thanks are due to Dr. C. Baffa for having written the first version of the control software for the instrument, afterwards developed and improved by Dr. K. Reardon; to G. Falcini and to S. Paloschi for having built the mechanical structure and the electronic controls; to T. Grisendi and F. Fabiani for assistance in assembling and testing the instrument; to Prof. A. Egidi, Prof. S. Cantarano and Prof. F. Berrilli who provided the software for the CCD acquisition system. Thanks are also due to Dr. A. Falchi, Prof. R. Falciani, and Dr. G. Cauzzi for their suggestions and encouragement.

IBIS was built with the contribution of the INAF - Osservatorio Astrofisico di Arcetri, the Università di Firenze, the Università di Roma "Tor Vergata", and the Ministero dell'Università e della Ricerca Scientifica.

Since June 2003 this instrument is installed at the Dunn Solar Telescope of the National Solar Observatory, operated by the Association of Universities for Research in Astronomy, Inc. (AURA), under cooperative agreement with the National Science Foundation. We are grateful to the observing staff of the DST for their continued assistance in the installation phase.

\section{References}

Allen, C.W.: 1985, Astrophysical Quantities. The Athlon Press, London.

Beckers, J.M.: 1998, Astron. Astrophys. Suppl. 129, 191.

Bendlin, C. and Volkmer, R.: 1995, Astron. Astrophys. Suppl. 112, 371.

Bendlin, C., Volkmer, R., and Kneer, F.: 1992, Astron. Astrophys. 257, 817.

Cavallini, F.: 1998, Astron. Astrophys. Suppl. 128, 589.

Cavallini, F., Berrilli, F., Cantarano, S., and Egidi, A.: 2000, in A. Wilson (ed.), The Solar Cycle and Terrestrial Climate, ESA Publications Division, Noordwijk, ESA SP-463, p. 607.

Kentischer, T.J., Schmidt, W., Sigwarth, M., and von Uexküll, M.: 1998, Astron. Astrophys. 340, 569.

Koschinsky, M., Kneer, F., and Hirzberger, J.: 2001, Astron. Astrophys. 365, 588.

Loughhead, R.E., Bray, R.J., and Brown, N.: 1978, Appl. Opt. 17, 415.

Neidig, D., Wiborg, P., Mozer, J., Dalrymple, N., Dunn, R., Gregory, S., and Gullixson, C.: 2003, Bull. Am. Astron. Soc. 35, 848.

Netterfield, R.P. and Ramsay, J.V.: 1974, Appl. Opt. 13, 2685.

Ramsay, J.V.: 1969, Appl. Opt. 8, 569.

Ramsay, J.V., Kobler, H., and Mugridge, E.G.V.: 1970, Solar Phys. 12, 492.

Rimmele, T.R., Richards, K., Hegwer, S.L. et al.: 2004, Proc. SPIE 5171, 179.

Steel, W.H.: 1967, Interferometry, Cambridge University Press, Cambridge, p. 125. 
Tritschler, A., Schmid, W., Langhans, K., and Kentischer, T.: 2002, Solar Phys. 211, 17. Vaughan, J.M.: 1989, The Fabry-Perot interferometer: History, Theory, Practice and Applications. Adam Hilger, Bristol and Philadelphia.

von der Lühe, O. and Kentischer, T.J.: 2000, Astron. Astrophys. 146, 499. 